\title{
Segmentation and Classification of Hyperspectral Images Using Watershed Transformation
}

\author{
Y. Tarabalka*,a,b, J. Chanussot ${ }^{\mathrm{a}}$, J.A. Benediktsson ${ }^{\mathrm{b}}$ \\ ${ }^{a}$ GIPSA-Lab - Grenoble Institute of Technology, \\ Domaine Universitaire - BP 46 - 38402 Saint-Martin-d'Hères Cedex, France \\ ${ }^{b}$ Faculty of Electrical and Computer Engineering - University of Iceland, \\ Hjardarhaga 2-6, 107 Reykjavik, Iceland
}

\begin{abstract}
Hyperspectral imaging, which records a detailed spectrum of light for each pixel, provides an invaluable source of information regarding the physical nature of the different materials, leading to the potential of a more accurate classification. However, high dimensionality of hyperspectral data, usually coupled with limited reference data available, limits the performances of supervised classification techniques. The commonly used pixel-wise classification lacks information about spatial structures of the image. In order to increase classification performances, integration of spatial information into the classification process is needed. In this paper, we propose to extend the watershed segmentation algorithm for hyperspectral images, in order to define information about spatial structures. In particular, several approaches to compute a one-band gradient function from hyperspectral images are proposed and investigated. The accuracy of the watershed algorithms is demonstrated by the further incorporation of the segmentation maps into a classifier. A new spectral-spatial classification scheme for hyperspectral images is proposed, based on the pixel-wise Support Vector Machines classification, followed by majority voting within the watershed regions. Experimental segmentation and classification results are presented on two hyperspectral images. It is shown in experiments that when the number of spectral bands increases, the feature extraction and the use of multidimen-
\end{abstract}

*Corresponding author. Tel.: +33 (0)6 669572 75; fax: +33 (0)4 76826384 . 
sional gradients appear to be preferable to the use of vectorial gradients. The integration of the spatial information from the watershed segmentation in the hyperspectral image classifier improves the classification accuracies and provides classification maps with more homogeneous regions, compared to pixel-wise classification and previously proposed spectral-spatial classification techniques. The developed method is especially suitable for classifying images with large spatial structures.

Key words: Hyperspectral images, mathematical morphology, watershed, segmentation, classification

\section{Introduction}

The growing availability of hyperspectral images has opened the door to numerous new applications in remote sensing and other areas of image analysis. Hyperspectral sensors capture more than a hundred spectral bands (data channels) simultaneously. Thus, each pixel in a hyperspectral image is presented as the vector of values corresponding to the wide spectrum of reflected light [1] (Figure 1 depicts the structure of a hyperspectral image). For instance, NASA Jet Propulsion Laboratory's Airborne Visible-Infrared Imaging Spectrometer (AVIRIS) system has 224 spectral channels with a spectral resolution of around $10 \mathrm{~nm}$, covering the wavelengths from 0.4 to $2.5 \mu \mathrm{m}[2]$. The fine spectral resolution of the data provides an invaluable source of information regarding the physical nature of the different materials, increasing the capability to distinguish structures and objects in the image scene.

However, such a large number of spectral channels implies the high dimensionality of the data and presents challenges to image analysis and classification. Most of the commonly used methods designed for the analysis of grey level, color or multispectral images are not appropriate for hyperspectral images. As a matter of fact, very limited reference data are usually available (the ratio of the number of referenced samples to the number of spectral channels quickly drops), which limits the performances of supervised classification techniques. 


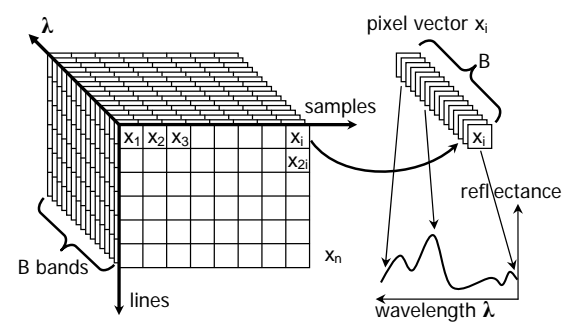

Figure 1: Structure of a hyperspectral image

Furthermore, for analysis of hyperspectral images the well known curse of dimensionality prevents robust statistical estimations, usual vector norms become meaningless and so on (e.g., the Hughes phenomenon [3]). Therefore, to take full advantage of the rich information provided by the spectral dimension, the development of new algorithms is required.

The first attempts to classify hyperspectral images were designed to assign each pixel to one of the classes based on its spectrum only [4]. These pixel-level processing systems use a wide range of features, such as the direct spectral information, texture features, and linear and nonlinear transformations of these features. The applied feature extraction procedure often aims at reducing the dimensionality of the data. The features are used for image classification with a wide range of techniques, such as maximum-likelihood or Bayesian estimation techniques $[5,6,7]$, neural networks $[8,9,10]$, decision trees $[11,12]$, genetic algorithms [13] and kernel-based methods [14, 15, 16, 17]. In particular, Support Vector Machines (SVM) and other kernel-based methods have recently shown good classification results, because they tend to be robust when a limited number of training samples is available.

Further modification to improve classification results consists in the integration of spatial and spectral information in the image analysis. It means that the decision to assign a pixel to a specific class is simultaneously based on the feature vector of this pixel and on some information derived from the pixel's neighborhood. In previous studies, the spatial context was included in 
the classification system by using morphological filters [15], morphological leveling [18] and Markov random fields [19]. These methods show promising results in terms of classification accuracies by incorporating spatial and spectral information. However, these algorithms use fixed-window-based neighborhoods. This involves the problem of scale selection, especially if the image contains some small or complex structures.

Another approach to define spatial structures consists in performing image segmentation $[20,21,22]$. The regions obtained from the obtained segmentation map define the spatial context of the pixels within these regions. To make this approach effective, an accurate segmentation of the image is needed.

In previous studies, several methods for multispectral image segmentation have been investigated. Numerous works exploit region merging techniques, where neighboring image segments are merged iteratively based mostly on their spectral similarity. For instance, the eCognition software performs multiresolution segmentation, based on bottom-up region merging [23]. Initially, each pixel is considered as a separate region, and subsequently pairs of regions are merged, based on a homogeneity criterion, which is a combination of spectral and shape properties. Tilton developed a hierarchical segmentation algorithm [24], which performs region growing and spectral clustering alternately. The main drawback of applying region merging for image segmentation is that the homogeneity criterion, or thresholds must be chosen. For accurate segmentation, these techniques usually produce a pyramid of segmentation maps, using a range of thresholds. Then, manual interpretation of the results is needed.

Other studies exploit mathematical morphology based segmentation approaches $[18,25,26,27,28,29,30]$, which mostly use granulometries or watershed transformation. The extension of morphological operators to the case of multispectral images is not straightforward, because there is no natural way for total ordering of multivariate pixels, which is a requirement in mathematical morphology. An extensive literature on mathematical morphology for color and multispectral images is available $[31,32,33,34,35,36]$. In particular, the watershed segmentation of color images was investigated in [37, 38, 39]. 
However, the above morphological and watershed methods are not suitable for segmentation of hyperspectral images, due to the following reasons:

- A hyperspectral image is composed of hundreds of spectral channels. Therefore, the use of total ordering schemes for multivariate data, such as the bit mixing paradigm [31], is not possible, because it would lead to a huge number of values stored for each pixel.

- In previous studies, polar-based representations (HLS, HSV) and perceptional color spaces (LUV, LAB) were used for morphological analysis of color images $[40,37]$. These methods are not applicable for hyperspectral images.

In a recent paper, Noyel et al. [29] gave an overview of the literature on the watershed-based multispectral image segmentation and performed watershed segmentation of hyperspectral images. Their method is composed of spectral classification to obtain markers and computation of a multivariate gradient to get spatial information. Only visual results (the obtained segmentation maps) are presented in the article. Therefore, the question of defining a watershed transformation for the case of hyperspectral images has only recently been raised in the literature and needs further investigations.

Some studies have been conducted on spectral-spatial classification of multispectral images. Linden et al. [41] used the vector mean as a feature for each region in a spectral-spatial classifier. First, they performed the segmentation of a hyperspectral image, based on region growing (using the eCognition software [23]). Then, a vector mean was computed for each region, such that the value in each band represented the average spectral information of the pixels in this region in the respective band. Afterwards, the regions were classified by an SVM classifier. However, the obtained results were mostly not an improvement over those obtained by the pixel-wise SVM classification. Li and Xiao [28] used spectral and spatial information for classification of a multispectral (4-band SPOT 5) image. There, a watershed segmentation and a pixel-wise 
maximum likelihood classification of an image were independently performed. Then, pixels of the whole region were assigned to one class if more than $50 \%$ of pixels in this region were categorized into one class by a pixel-wise classifier. The classification results were substantially improved with the spectral-spatial approach compared to the pixel-wise maximum likelihood classification.

Widayati et al. [42] used spatial information in a classifier, in order to perform a spectral-spatial classification of a multispectral (4-band IKONOS) image. First, a segmentation map was obtained using the Merge Using Moments algorithm [43]. Then two options were explored. First, each region from the segmentation map was classified using its vector mean as a feature. In another approach, the pixel-wise classification map obtained by maximum likelihood classifier was combined with the segmentation map using majority voting: for every region from the segmentation map, all the pixels were assigned to the majority class within this region. Results of the pixel-wise maximum likelihood classification were also used for comparison. Of all these three methods, the spectral-spatial classification using majority voting gave the highest overall accuracy.

The main contributions of this paper are two-fold:

1. The first contribution is the extension of the watershed segmentation algorithm to hyperspectral data. Watershed transformation is usually applied to the gradient, which must be a scalar function, in order to form a complete lattice structure [44]. When processing a hyperspectral image, information about spatial structures must be extracted from all bands in the optimal way. Different approaches to segment the hyperspectral image by watershed are proposed and investigated. In particular, different ways to compute a one-band gradient function of a hyperspectral image are considered. We emphasize that this study is not a comparison of watershed segmentation methods in general. Instead, it focuses on the extension of watershed transformation for hyperspectral images, for the purpose of accurate segmentation and further classification. 
2. The obtained watershed segmentation map is further incorporated into a spectral-spatial classifier, aiming at improving classification accuracies, when compared to pixel-wise classification. Thus, the second contribution of this paper is the development of the new segmentation and classification scheme to analyze hyperspectral data. The proposed classification method combines results of a pixel-wise SVM classification and the segmentation map using majority vote approach [21]. This contribution can be summarized as follows: the segmentation defines an adaptive neighborhood for each pixel which is used for the spatial regularization following a pixel-wise classification.

To test the developed segmentation and classification algorithms, two hyperspectral airborne images are used: A 103-band ROSIS (Reflective Optics System Imaging Spectrometer) image of the University of Pavia, Italy, and a 220-band AVIRIS image taken over the Northwestern Indiana's Indian Pine site [45].

The paper is organized as follows. In Section 2, an overview of the watershed technique is given, and then the extension of the watershed algorithm to hyperspectral images is discussed. In Section 3, the developed segmentation and classification scheme is presented. In Section 4, experimental results and comparisons are presented and discussed. Finally, conclusions are drawn in Section 5 .

\section{Watershed segmentation}

Watershed transformation is a powerful mathematical morphology technique for image segmentation $[46,44]$. It was introduced in image analysis by Beucher and Lantuéjoul [47], and subsequently a lot of algorithms for its implementation have been proposed.

The watershed transform considers a two-dimensional one-band image as a topographic relief. The value of a pixel $h$ stands for its elevation. The watershed lines divide the image into catchment basins, so that each basin is associated with one minimum in the image (see Fig. 2). The watershed transformation 


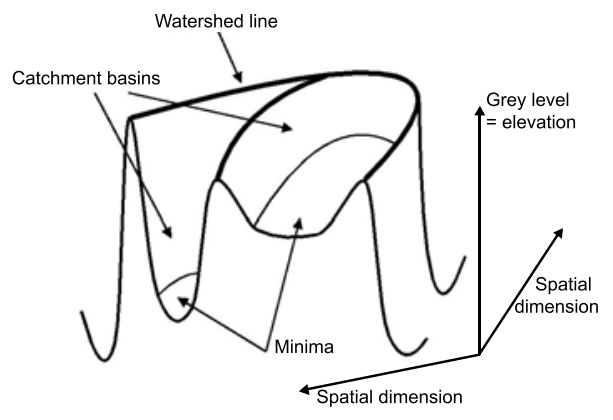

Figure 2: Topographic representation of a one-band image

is usually applied to the gradient function of the image. The gradient defines transitions between regions, so that it has high values on the borders between objects and minima in the homogeneous regions. And in this case, if the crest lines in the gradient image correspond to the edges of image objects, watershed transformation partitions this image into meaningful regions.

A wealth of literature describes techniques for computing the watershed transformation (see for instance $[48,49,50,51]$ ). A review of watershed algorithms can be found in $[48,50]$. Vincent and Soille [48] have proposed an efficient watershed algorithm using flooding simulations, which has become one of the classical algorithms to compute watersheds.

The output of the watershed transform is a partition of the image composed of regions (sets of pixels connected to the same local minimum) and of watershed pixels (WHEDs, the borders between the regions). Figure 3 shows an example of watershed transformation in one dimension, where three regions, associated with the three minima, are defined. The two maxima correspond to the borders between regions and are not assigned to any region (watershed pixels).

Typically, the result of watershed segmentation on the gradient image without any additional processing is a severe oversegmentation (every single local minimum of the gradient leads to one region). Common ways to reduce the number of local minima are to filter the original image or the gradient function (e.g., area filtering) or to use markers [46]. The oversegmentation effect 


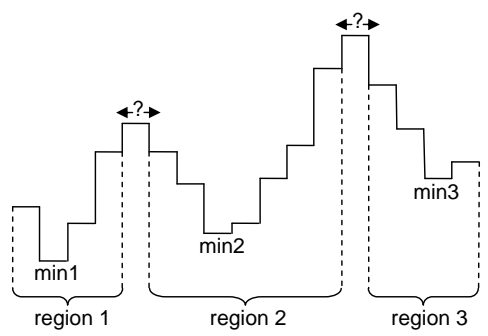

Figure 3: Example of watershed transformation in one dimension.

can be also corrected using some post-processing, such as merging of similar neighboring regions.

In the next subsection, different approaches for segmentation of hyperspectral images by watershed are discussed and extended.

\subsection{Watershed segmentation of hyperspectral images}

As previously mentioned, the watershed transformation requires as input a one-band image and gives as a result a one-band segmentation map (where each pixel contains the label of the catchment basin or the watershed pixel label). In this paper, we aim to apply this transformation to a $B$-band hyperspectral image. Let us consider this image as a set of $n$ pixel vectors $\mathbf{X}=\left\{\mathbf{x}_{j} \in \mathbb{R}^{B}, j=\right.$ $1,2, \ldots, n\}$ (each pixel is characterized by its spatial location and a vector of spectral values; see Fig. 1). We denote also the image of every spectral band as $X_{\lambda}, \lambda=1,2, \ldots, B$. Different strategies are possible to compute watersheds. They are summarized in Figure 4.

Before computing a gradient, feature extraction on the original image can be performed, applying one of the transformations such as the Principal Component Analysis (PCA) [52, 53], Maximum Noise Fraction (MNF) [54] and Independent Component Analysis (ICA) $[55,56]$. The aim of this step is to obtain either a one-band image or a multi-band image which would contain enough information to distinguish between spatial structures in the image.

If a one-band image with a good distinguishing capability between structures can be obtained, the algorithm for computing a gradient and watershed 


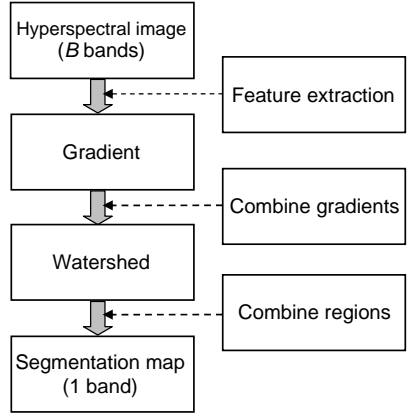

Figure 4: Flow-chart which shows strategies of applying watershed to a hyperspectral image

is straightforward. For instance, for a one-band image $Y$, a basic morphological gradient (also called the Beucher gradient) can be applied. It is defined as the arithmetic difference between the dilation and the erosion of $Y$ by the structuring element $E[44]$ :

$$
\rho_{E}(Y)=\delta_{E}(Y)-\varepsilon_{E}(Y)
$$

If, however, at the input of the gradient step we still have a multi-band image, we can proceed in different ways, that can be grouped into the following three categories:

- to compute a vectorial gradient;

- to compute a multidimensional gradient;

- to combine watershed segmentation maps a posteriori.

These three options are discussed in the next three subsections.

\subsubsection{Computation of a vectorial gradient}

Vectorial gradients are based on the distance between pixel vectors, and produce from the $B$-band image one-band gradient $[57,29]$. Several types of vectorial gradients have been proposed. Noyel et al. [29] proposed to use a metric-based gradient for hyperspectral images, which is described as follows: 
For each pixel vector $\mathbf{x}_{p}$, let $\psi=\left[\mathbf{x}_{p}^{1}, \mathbf{x}_{p}^{2}, \ldots, \mathbf{x}_{p}^{e}\right]$ be a set of $e$ vectors in the neighborhood of $\mathbf{x}_{p}$ (set $\psi$ does not contain $\mathbf{x}_{p}$ ). For instance, a four- or an eight-neighborhood ( $e=4$ or $e=8$, respectively) can be used. The metric-based gradient is defined as a difference between the supremum and the infimum of the defined distances between $\mathbf{x}_{p}$ and vectors from the set $\psi$ :

$$
\nabla_{\psi, d}^{M B}\left(\mathbf{x}_{p}\right)=\sup _{i \in \psi}\left\{d\left(\mathbf{x}_{p}, \mathbf{x}_{p}^{i}\right)\right\}-\inf _{j \in \psi}\left\{d\left(\mathbf{x}_{p}, \mathbf{x}_{p}^{j}\right)\right\} .
$$

Various distances can be used to compute gradient from (2) such as Euclidean, Mahalabobis, chi-squared distances [29].

Another type of vectorial gradient is the Robust Color Morphological Gradient (RCMG). This gradient was developed for color images by Evans and Liu [57]. Here we investigate the use of the RCMG for hyperspectral images.

For each pixel vector $\mathbf{x}_{p}$, let $\chi=\left[\mathbf{x}_{p}^{1}, \mathbf{x}_{p}^{2}, \ldots, \mathbf{x}_{p}^{e}\right]$ be a set of $e$ vectors within a structuring element $E$, which defines the neighborhood of the vector $\mathbf{x}_{p}$, and the set $\chi$ contains $\mathbf{x}_{p}$. The Color Morphological Gradient (CMG) is computed as

$$
\nabla_{\chi, d}^{C M}\left(\mathbf{x}_{p}\right)=\max _{i, j \in \chi}\left\{d\left(\mathbf{x}_{p}^{i}, \mathbf{x}_{p}^{j}\right)\right\}
$$

i.e., the maximum of the distances between all pairs of vectors in the set $\chi$. Here, various distances can be chosen. If the Euclidean distance is used, (3) can be rewritten as

$$
\nabla_{\chi, d}^{C M}\left(\mathbf{x}_{p}\right)=\max _{i, j \in \chi}\left\{\left\|\mathbf{x}_{p}^{i}-\mathbf{x}_{p}^{j}\right\|_{2}\right\}
$$

One of the drawbacks of the CMG is that it is very sensitive to noise. To overcome the problem of outliers, the authors of [57] have proposed to use the RCMG. The scheme to make the CMG robust consists in removing the two pixels that are the furthest apart and then finding the CMG of the remaining pixels. This process can be repeated several times until a good estimate of the gradient is obtained. Thus, the $R C M G$, using the Euclidean distance, can be defined as

$$
\nabla_{\chi, d}^{R C M}\left(\mathbf{x}_{p}\right)=\max _{i, j \in\left[\chi-R E M_{r}\right]}\left\{\left\|\mathbf{x}_{p}^{i}-\mathbf{x}_{p}^{j}\right\|_{2}\right\}
$$


where $R E M_{r}$ is the set of the $r$ vector pairs removed. The appropriate value of the parameter $r$ in (5) depends on the chosen structuring element $E$ and the amount of noise present in the image, as discussed in [57]. When a oneband vectorial gradient is computed, it is used as the input of the watershed algorithm.

\subsubsection{Multidimensional gradient methods}

Another approach to compute a one-band gradient from the multi-band image consists in considering the $B$-band image as a set of $B$ one-band images. In this case, the gradients of every spectral band can be computed, using for instance a morphological gradient (see (1)). Then the obtained $B$ gradient images $\rho_{E}\left(X_{\lambda}\right), \lambda=1,2, \ldots, B$ are combined into one image using linear or non-linear operators.

As an example of the linear operators, the weighted sum of gradients can be computed by

$$
\nabla_{E}^{+}(\mathbf{X})=\sum_{\lambda=1}^{B} \omega_{\lambda} \rho_{E}\left(X_{\lambda}\right),
$$

where $\omega_{\lambda}$ denotes the weight of the gradient of the band $\lambda$. If $\omega_{\lambda}=1, \lambda=$ $1,2, \ldots, B$, all the bands are supposed to have an equal importance in defining the gradient. Modifying the weight coefficients, the gradient estimation can be improved. For instance, coefficients that are inversely proportional to the estimated noise of each spectral band can be used as the weights in (6).

Examples of non-linear operators are the supremum and the median operators. The gradient supremum over morphological gradients of every band is defined as follows: for every pixel the supremum over all gradient images is taken as the output value of this pixel.

\subsubsection{Combination of watershed segmentation maps}

First, $B$ standard gradients are computed, one for each spectral band. Then each gradient image is used to compute a watershed transformation. This gives $B$ segmentation maps that can be further combined to provide a single output segmentation map. 
One of the ways to combine the $B$ segmentation maps, in order to define relevant edges, consists in summing the watershed lines. Here, for each segmentation map obtained from the gradient of band $\lambda$, a binary image $W_{\lambda}$ of watershed lines is produced. Thus, $W_{\lambda}$ is an image in which watershed pixels are equal to 1 and all other pixels are equal to 0 . The sum of watershed lines is computed by

$$
\mathbf{W}=\sum_{\lambda=1}^{B} W_{\lambda} .
$$

The obtained image $\mathbf{W}$ can be further thresholded, in order to define the border pixels that were presented in most of the segmentation maps, hence ensuring a reliable edge detection.

However, when summing the watershed lines, we do not have information about regions anymore, but only about edges. Furthermore, some edges can become open after thresholding. Therefore, closing of edges and image region labeling must be performed after the procedure described above.

\section{Segmentation and classification scheme}

As previously mentioned, the information about spatial structures defined by the watershed segmentation algorithm can be used to improve the results of classification of a hyperspectral image. In this section, a new combined spectral-spatial classification scheme is presented for hyperspectral images based on watershed segmentation.

The general flow-chart of the proposed segmentation and classification scheme is given in Figure 5. At the input we have a $B$-band hyperspectral image $\mathbf{X}=\left\{\mathbf{x}_{j} \in \mathbb{R}^{B}, j=1,2, \ldots, n\right\}$. First, the watershed segmentation is performed on this image, using one of the approaches discussed in Section 2 and summarized in Figure 4. In the resulting segmentation map, each pixel contains the label of the region it belongs to, or the watershed pixel label (see Figures 3,6).

It is often desirable to produce a segmented image where each pixel belongs to some region, without border pixels between regions. In this case, each watershed pixel can be assigned to one of the regions in its neighborhood. For this pur- 


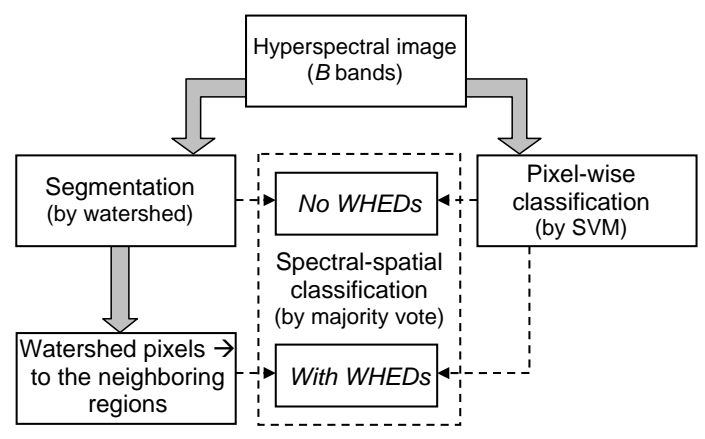

Figure 5: Flow-chart of the proposed segmentation and classification scheme.

pose, we propose to compute for every region $\mathbf{S}\left(\mathbf{S}=\left\{\mathbf{s}_{j} \in \mathbb{R}^{B}, j=1,2, \ldots, l\right\}\right.$, $\mathbf{S} \subseteq \mathbf{X}$, with $l$ equal to the number of pixels in the region) the standard vector median [58]. A standard vector median $\mathbf{s}_{V M}$ for a set of pixel vectors is a vector, which fulfills the condition that the sum of the distances between this vector and all the other vectors in the set is minimal (for instance, $L_{1}$ norm is used to compute distances):

$$
\mathbf{s}_{V M}=\arg \min _{\mathbf{s} \in \mathbf{S}}\left\{\sum_{j=1}^{l}\left\|\mathbf{s}-\mathbf{s}_{j}\right\|_{1}\right\} .
$$

Every watershed pixel is assigned to the neighboring region with the "closest" median, i.e., the distance between the vector median of this region and the watershed pixel vector is minimal (see the example in Fig. 6).

After the image is segmented into regions, this spatial information should be used to improve the classification results. Two approaches to integrate spectral and spatial information into the classification system can be distinguished:

1. To define a feature or a set of features for each region from the segmentation map, and classify regions using these features.

2. To perform a pixel-wise classification first, and then combine a pixel-based classified image with the segmentation results.

Here we propose to use the second approach for the spectral-spatial classification of hyperspectral images, in order to improve the results of the pixel- 


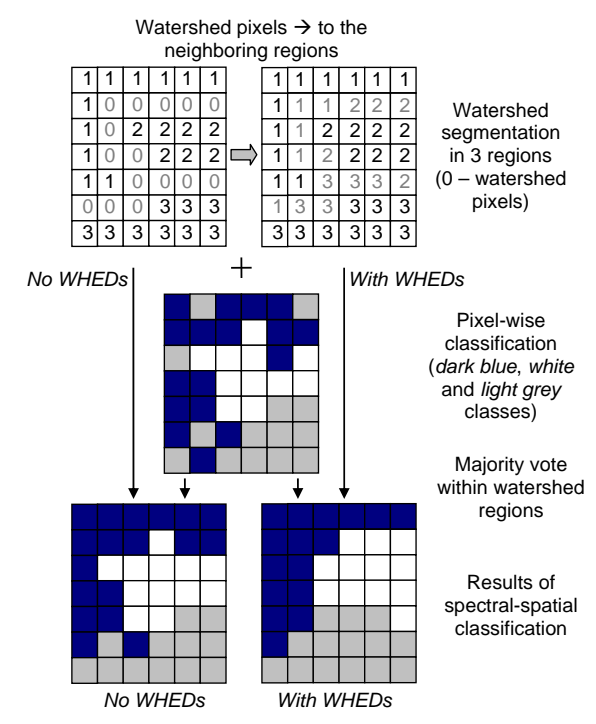

Figure 6: Example of spectral-spatial classification.

wise classifier using the spatial information from the segmentation map (see Figures 5,6$)$. The proposed approach is outlined in [21]. First, a pixel-wise classification by SVM on the original hyperspectral image is performed. Then, for every watershed region $\mathbf{S}$, all the pixels are assigned to the most frequent class within this region (majority vote approach). Please note that majority vote within fixed neighborhoods is a standard spatial regularization procedure following a pixel-wise classification. Here, we propose to use the results of a segmentation to define an adaptive neighborhood for each pixel.

The watershed pixels can be either left not processed during the majority voting (what we call the No WHEDs approach), or assigned to the regions with the "closest median" before the majority voting is performed considering all the pixels (what we call the With WHEDs approach). Figure 6 shows an example of the combination of the pixel-wise classification map and the segmentation map by majority vote, using both No WHEDs and With WHEDs approaches. 


\section{Experimental results and discussion}

\subsection{Segmentation and classification of the University of Pavia image 4.1.1. Dataset}

The University of Pavia image is of an urban area that was recorded by the ROSIS-03 optical sensor. The image scene is the urban area surrounding the University of Pavia, Italy. The image has spatial dimensions of 610 by 340 pixels, with a spatial resolution of $1.3 \mathrm{~m}$ per pixel. The number of bands of the ROSIS03 sensor is 115 with a spectral coverage ranging from 0.43 to $0.86 \mu \mathrm{m}$. The 12 most noisy channels have been removed, and the experiments are conducted on the 103-band image. Nine classes of interest are considered, with the number of test and training samples detailed for each class in Table 2. Training-test set was provided by Prof. Paolo Gamba together with the hyperspectral image. False color image of the University of Pavia and the reference data are presented in Figure 7.

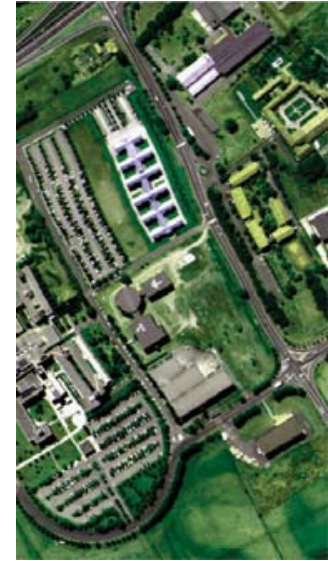

(a)

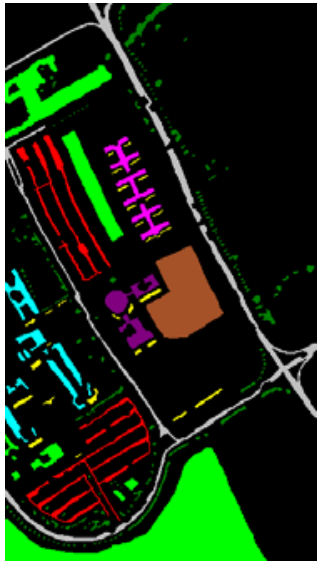

(b)

Figure 7: University of Pavia image: (a) Three-band color composite; (b) Reference data: Asphalt, meadows, gravel, trees, metal sheets, bare soil, bitumen, bricks, shadows and nonlabeled pixels.

\subsubsection{Experimental results}

Different approaches of the watershed transformation of hyperspectral images described in Section 2 were tested. Watershed segmentation was performed 
on the gradient function obtained in four different ways:

1. Band50: A morphological Beucher gradient (see (1)) was computed on one band. Band No. 50 was chosen arbitrarily, but similar results were obtained with other non noisy bands. Here and in all the following morphological operations, a $3 \times 3$ square structuring element $E$ was used (center of $E$ was in the center of the square).

2. SumBands: First, a morphological gradient for every band was computed. Then, the sum of gradients was obtained by (6), assuming that $\omega_{\lambda}=1$, $\lambda=1,2, \ldots, B$.

3. Sum $4 P C A$ : First, the PCA transformation was performed on the original image (using the ENVI software [59]). The first four principal components contained $99.16 \%$ of the total variance in the data. Morphological gradients of the first four PCA components were computed, and then summed together (using (6), with equal unitary weights).

4. $R C M G$ : The RCMG on the original image was computed by (5), with $r=1$.

The obtained four gradient images are shown in Figure 8. The principal borders of objects are defined by all gradients. Thus, most of the spatial structures can be recognized only from the single band No. 50. However, some structures, for instance shadows were not defined by the gradient Band50 (see Fig. 8.(a), left-bottom corner of the image). The borders of shadows are present in the other three gradient images, as in this case the information from all bands was used for computation. If we compare the images of gradients SumBands and Sum 4 PCA (Figures 8.(b) and 8.(c), respectively), the latter one seems to be less noisy (for instance, see the meadows part in the bottom of the image). Also, the Sum 4 PCA gradient defines roads well. But as the four PCA components do not contain all the information in the original image, some details are missed in the Sum $4 P C A$ gradient image (for instance, trees). The $R C M G$ gradient (see Fig. 8.(d)) defines borders that are thinner and clearer than those defined by the other three gradients. 


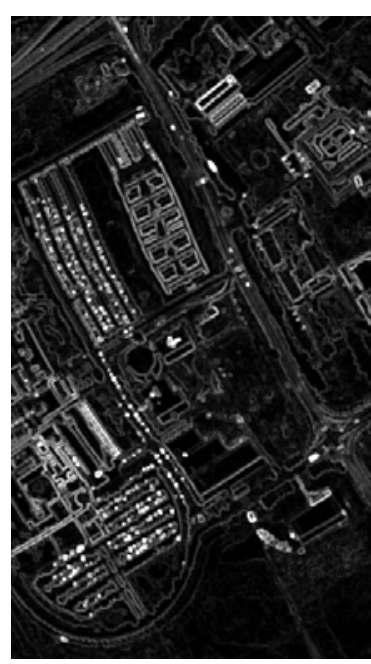

(a)

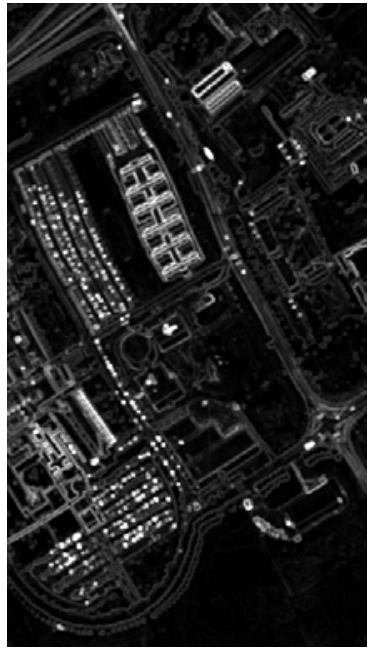

(c)

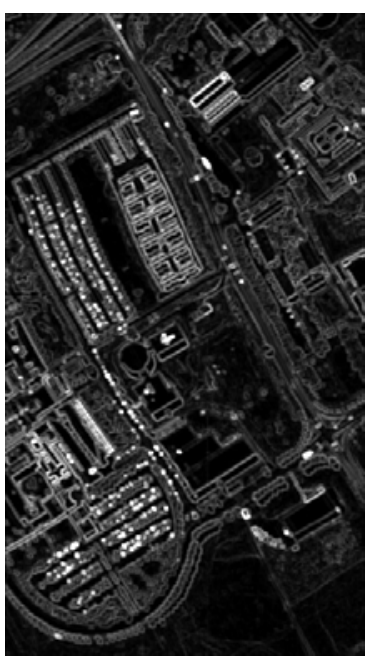

(b)

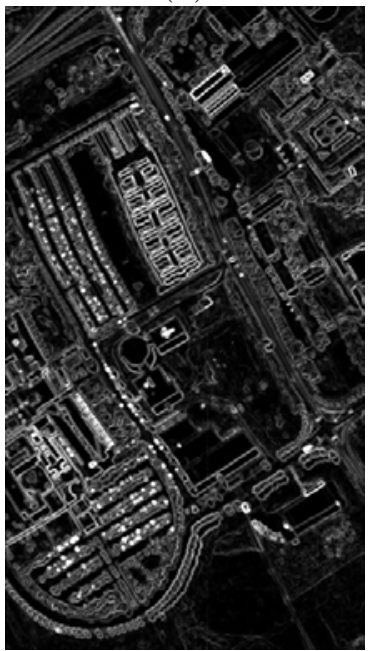

(d)

Figure 8: Gradients of the University of Pavia image: (a) Gradient of band No. 50; (b) Sum of gradients over all bands; (c) Sum of gradients for the first four PCA components; (d) RCMG using the Euclidean distance, $r=1$. 


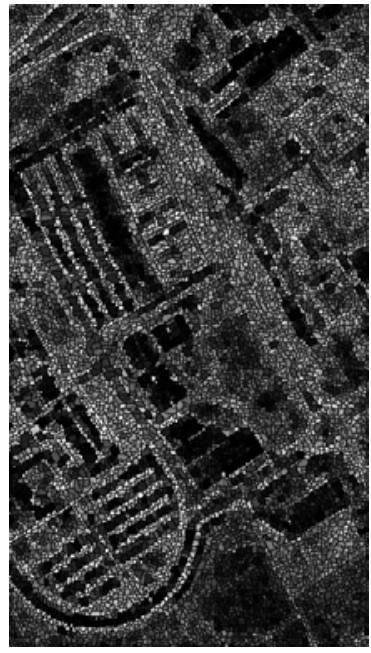

(a)

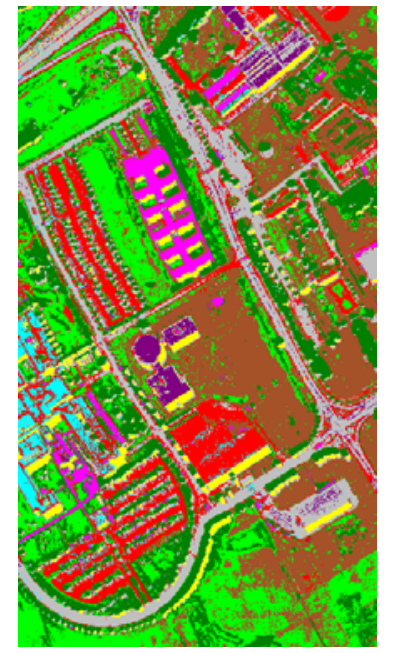

(b)

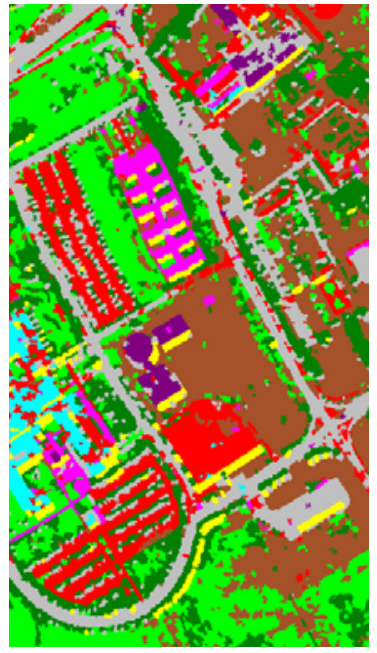

(c)

Figure 9: University of Pavia image: (a) Watershed on the $R C M G$, using the Euclidean distance, $r=1$; (b) Classification map for the SVM classification; (c) Classification map for the spectral-spatial classification (using watershed map on the RCMG, With WHEDs approach).

Furthermore, the watershed transformation was applied to each of the four obtained gradients, using the algorithm of Vincent and Soille [48] (based on 8neighborhood connectivity). The resulting segmentation maps for watershed on Band50, SumBands, Sum $4 P C A$ and $R C M G$ gradients contained 11641, 10558, 10345 and 11802 regions, respectively. Figure 9.(a) shows the segmentation map based on the $R C M G$, where the main spatial structures can be seen. (The colors of each region correspond to the label of this region, scaled in order to obtain a grey-scale 8-bit image.)

As expected, the obtained watershed results were severely oversegmented. Objects were represented mostly by several regions. The first aim was to obtain the segmentation map where each region contained pixels belonging to one object, i.e., where there were no undersegmentation errors. As mentioned in Section 2, oversegmentation can be corrected by merging regions. On the other hand, it may be more difficult to cope with the undersegmentation problem. 
To assess qualitatively the accuracy of the watershed segmentation, the obtained segmentation maps were incorporated into the combined spectral-spatial classifier. First, for every segmentation map, the vector median of every region was computed, and the watershed pixels were assigned to one of the neighboring regions based on the minimal distance between the watershed pixel and the vector median of the different regions.

The multi-class pairwise (one versus one) SVM classification, with the Gaussian Radial Basis Function (RBF) kernel, of the original hyperspectral image was performed, using the LIBSVM library [60]. The optimal parameters $C$ and $\gamma$ were determined by 5 -fold cross validation: $C=128, \gamma=0.125$. Figure 9.(b) shows the obtained classification map.

After the pixel-wise SVM classification, the majority vote within the watershed regions was performed. Both the No WHEDs and With WHEDs approaches introduced in Section 3 were applied for each of the four segmentation maps. The global classification accuracies for the pixel-wise SVM and combined spectral-spatial classification are presented in Table 1. Here and in the following the accuracies are based on results for test data. The following measures of accuracy were used:

- Overall Accuracy (OA) is the percentage of correctly classified pixels;

- Average Accuracy (AA) is the mean of class-specific accuracies, i.e., the mean of the percentage of correctly classified pixels for each class;

- kappa coefficient $(\kappa)$ is the percentage of agreement (correctly classified pixels) corrected by the number of agreements that would be expected purely by chance [52].

The number of training and test samples along with the class-specific accuracies for test data are given in Table 2. Figure 9.(c) shows the classification map for the spectral-spatial classification, using the watershed map on the $R C M G$ and the With WHEDs approach. In order to compare the obtained results with previous works that used an SVM and spatial information for hyperspectral image 
classification, we have included in Tables 1 and 2 accuracies of mathematical morphology-based classification of the University of Pavia image using an SVM, principal components and extended morphological profiles (EMP); results are taken from Plaza et al. [61], where the same training and test samples were used for classification. This method was recently proposed by Benediktsson et al. [62] and is considered as one of the most advanced methods for spectralspatial classification of a multi-band data. Other results of joint spectral-spatial classification of the considered image can be found in $[63,15,64]$.

Table 1: Global classification accuracies in percentage for the University of Pavia image: Overall Accuracy (OA), Average Accuracy (AA) and kappa coefficient $(\kappa)$.

\begin{tabular}{|c|c|c|c|c|c|}
\hline \multicolumn{3}{|c|}{ Method } & $\mathrm{OA}$ & $\overline{\mathrm{AA}}$ & $\kappa$ \\
\hline \multicolumn{3}{|c|}{ Pixel-wise SVM } & 81.01 & 88.25 & 75.86 \\
\hline \multirow{8}{*}{$\begin{array}{c}\text { SVM + } \\
\text { Majority Vote }\end{array}$} & \multirow{4}{*}{ No WHEDs } & Band50 & 83.00 & 89.59 & 78.31 \\
\hline & & SumBands & 83.10 & 89.80 & 78.44 \\
\hline & & Sum4PCA & 83.36 & 89.85 & 78.76 \\
\hline & & RCMG & 83.48 & 90.03 & 78.89 \\
\hline & \multirow{4}{*}{ With WHEDs } & Band50 & 84.83 & 90.68 & 80.57 \\
\hline & & SumBands & 85.04 & 90.73 & 80.83 \\
\hline & & Sum4PCA & 85.35 & 91.09 & 81.23 \\
\hline & & $\mathrm{RCMG}$ & 85.42 & 91.31 & 81.30 \\
\hline \multicolumn{3}{|c|}{ EMP } & 85.22 & 90.76 & 80.86 \\
\hline
\end{tabular}

As can be seen from Table 1, all the SVM-based classifiers give high classification accuracies. Furthermore, the combining of spatial information obtained by watershed segmentation with the spectral classification results improves substantially the classification accuracies. The With WHEDs approach gave the best accuracies for all four segmentation results. The best global accuracies are achieved when performing the spectral-spatial classification based on the segmentation map on the RCMG and applying the With WHEDs approach. In that case, the overall accuracy is improved by 4.41 and the average accuracy is improved by 3.06 percentage points compared to the pixel-wise SVM classification. The integration of the different segmentation results in the classification improves the accuracies differently: SumBands performs better than Band50, Sum $4 P C A$ better than SumBands, and RCMG performs best. 


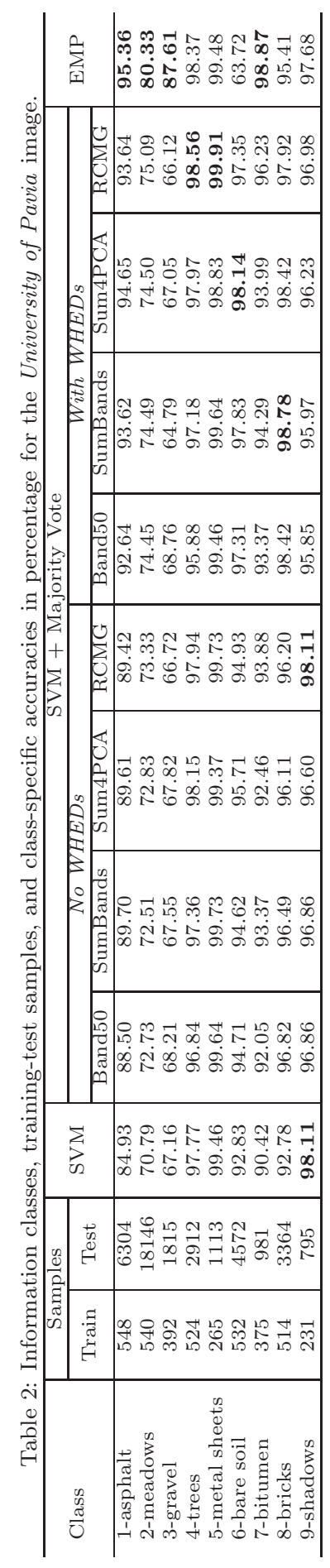


Classification accuracies were improved by the spectral-spatial classification for almost all the classes (see Table 2). For some classes, like alphalt, bitumen and bricks, accuracies were much improved by including spatial information, i.e., the accuracies improved by 5.81 to 9.72 percentage points, mostly because of the noise reduction. Here, different segmentation approaches led to the best classification accuracies for different classes. The $R C M G$ segmentation map improved the classification accuracies for the classes meadows, trees, metal sheets and bitumen. For the classes alphalt and bare soil, the best accuracies were achieved when applying the Sum $4 P C A$ segmentation. That confirms the assumption that the Sum 4 PCA gradient defined well the information about road structures. For the classes gravel and bricks, the best accuracies were achieved using the Band50 and SumBands approaches, respectively.

\subsubsection{Concluding discussion}

Based on the above, the following conclusions can be drawn about the accuracy of the watershed segmentation:

1. The $R C M G$ leads to the best segmentation and classification results. Applying the vectorial gradient based on the Euclidean distance between pixel vectors to the hyperspectral image gave the best classification accuracies, despite the high-dimensionality of data.

2. The watershed segmentation based on the gradient of one band is the least accurate approach as was expected, since it may be difficult to distinguish some different neighboring structures when using only one particular band. Still, as the inclusion of the information from the Band50 segmentation map improved the classification accuracies, most of the spatial structures could be retrieved from this single band.

3. The summing of the gradients of the first four PCA components gave slightly better results than the summing of the gradients of all bands, which indicates that the first PCA components contain the most important spatial information. Thus, applying the feature extraction before 
computing the gradient and watershed leads to better segmentation results.

Thus, the incorporation of spatial information into the classifier using the majority vote approach led to more homogeneous objects in the resulting classification map, when compared to the pixel-wise classification. However, when performing watershed segmentation, it is usually difficult to identify small but significant structures as separate regions. They may be identified as the border pixels and then be assimilated with one of the neighboring regions. Therefore, the classification accuracies of small classes, in our case trees and shadows, are not improved significantly, or reduced.

These conclusions are confirmed by visual inspection, when comparing the classification maps of the pixel-wise versus spectral-spatial classification (see Figures 9.(b) and 9.(c)). The spectral-spatial classification reduces significantly the noise in the classification map, resulting in more homogeneous regions in the output map. It can be also seen on the left-bottom part of the image that some small shadows regions were assimilated with the regions in their neighborhood.

When we compare the obtained results with the recent results of spectralspatial classification using SVM and EMPs (see Tables 1 and 2), the proposed segmentation and classification approach leads to higher global accuracies. Furthermore, accuracies for 4 from 9 classes are improved by our technique.

\subsection{Segmentation and classification of the Indiana image}

\subsubsection{Dataset}

In the second case study, the developed segmentation and classification algorithms are tested on a hyperspectral image of a rural area (the Indiana image) with more bands and a lower spatial resolution as compared to the University of Pavia image. The Indiana image was captured by the AVIRIS sensor over the Indian Pine test site in Northwestern Indiana [45]. The image is 145 by 145 pixels, and the spatial resolution is $20 \mathrm{~m}$ per pixel. It is composed of 220 spectral channels, and the full spectral range was used for experiments. The data contains 16 classes, which are detailed in Table 4, with a number of samples for 
each class in the available reference data. Figure 10 depicts the 3-band false color composite and the reference data. In our experiments, we have chosen randomly $10 \%$ of the samples for each class from the reference data as training samples (in order to test classification performances when limited reference data are available).

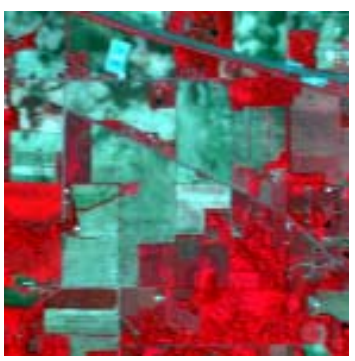

(a)

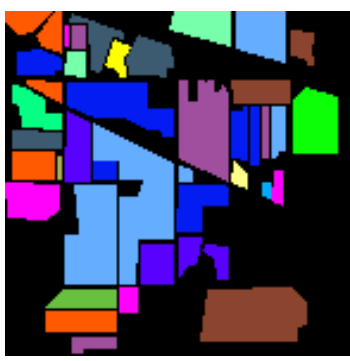

(b)

Figure 10: Indiana image: (a) Three-band color composite (bands 50, 27, 17); (b) Reference data: Corn-no till, Corn-min till, Corn, Soybeans-no till, Soybeans-min till, Soybeans-clean till, , Grass/pasture, Grass/trees, Grass/pasture-mowed, Hay-windrowed, Oats, Wheat, Woods, Bldg-Grass-Tree-Drives, Stone-steel towers and non-labeled pixels.

\subsubsection{Experimental results}

In this experiment, we do not choose one single band to perform a gradient, because there are more classes, with similar spectral responses, and some classes can be confused using only one specific band. Thus, three gradient functions were computed: SumBands, Sum $4 P C A, R C M G$. They were computed in the same way as for the University of Pavia image, as described in the previous subsection. For the Indiana image, the first four PCA components contain $99.23 \%$ of the total variance for the data.

As for the previous data set, the watershed transformation was applied to each of the gradient functions. The segmentation maps contain 1215, 1097 and 1277 regions for the SumBands, Sum $4 P C A$ and $R C M G$ gradients, respectively. Figure 11.(a) shows the segmentation map obtained from the SumBands gradient. It is difficult to evaluate the accuracy of segmentation from the image, since it is strongly oversegmented. In order to perform such an evaluation, 


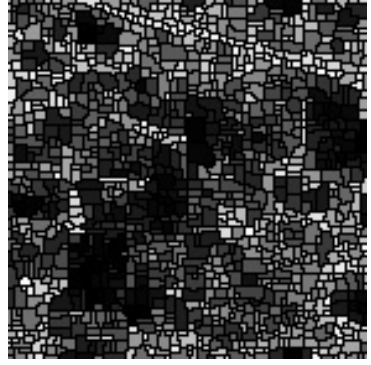

(a)

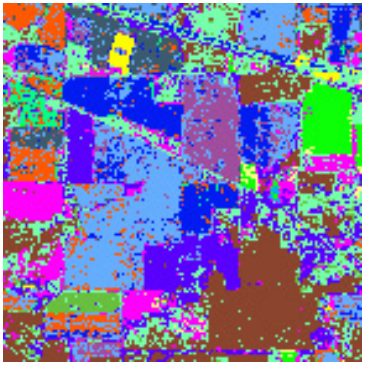

(b)

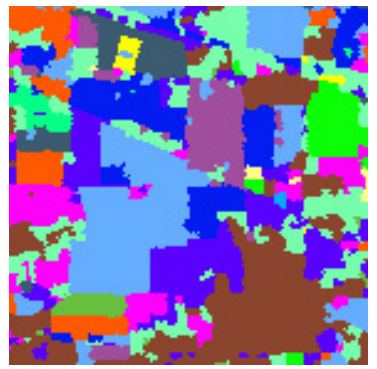

(c)

Figure 11: Indiana image: (a) Watershed on the sum of gradients over all bands; (b) Classification map for the SVM classification; (c) Classification map for the spectral-spatial classification (using SumBands method, With WHEDs approach).

the information from the segmentation maps was used for the spectral-spatial classification, as described below.

First, the segmentation maps for the With WHEDs approach were computed, by assigning the watershed pixels to the neighboring regions that had the closest vector median. Then, the multi-class one versus one SVM classification, with the Gaussian RBF kernel, of the original Indiana image was performed. The parameters $C$ and $\gamma$ were computed by 5 -fold cross validation giving: $C=$ $1024, \gamma=2^{-7}$. The resulting classification map is given in Figure 11.(b). The classification accuracies for test samples are shown in Tables 3 and 4.

The low spatial resolution of the Indiana image leads to the presence of highly mixed pixels which complicates the classification problem. Furthermore, some classes represent small crop fields, and the number of samples in the reference data for different classes varies from 20 to 2468 samples per class. To train the SVM classifier, $10 \%$ of the samples for each class were chosen randomly from the reference data. Therefore, some classes were represented by only a few samples in the training set (2 samples for the class oats), which may not provide sufficient representatives for the small classes.

Despite these problems, $78.76 \%$ of the pixels in the test set were correctly classified by the SVM classifier. As expected, the classification accuracies for the classes that were represented by only a few training samples are low (for 
instance, only $22.22 \%$ accuracy was observed for the class oats ). The low classification accuracies for the classes alfalfa, grass/pasture-mowed and oats reduced the average classification accuracy to $69.66 \%$.

Now we turn to the spectral-spatial classification. As in the first experiment, the watershed segmentation maps were used in the spectral-spatial classification based on the majority vote method, with the No WHEDs and With WHEDs approaches.

Tables 3 and 4 give global and class-specific classification accuracies for the spectral-spatial classification of the Indiana image, respectively. Figure 11.(c) shows the classification map for the spectral-spatial classification, using the watershed map on the SumBands gradient and the With WHEDs approach. Previous classification results for the Indiana image can be found in [65, 61] for comparison. However, the accuracies in the referenced works are not directly compared with those given in this paper, because different training-test sets are used. Furthermore, in order to evaluate the efficiency of the proposed segmentation and classification scheme using watershed, we have included in Tables 3 and 4 the spectral-spatial classification results, obtained by applying another segmentation technique followed by the procedure proposed in this paper (SVM classification and majority vote within the regions from a segmentation map). This time, the Hierarchical Image Segmentation (HSEG) algorithm [24] was used to segment a hyperspectral image. The NASA Goddard's RHSEG software provides an efficient implementation of this algorithm [66], which we used for our investigation. At the initialization step each pixel is considered as one region. The algorithm merges iteratively the most similar adjacent and nonadjacent regions. We used a Spectral Angle Mapper between the region mean vectors as the dissimilarity criterion between regions. The relative importance of merging of non-adjacent regions versus region growing (when only adjacent regions are merged) can be tuned. From experimental results, simple region growing leads to the highest classification accuracies. Segmentation maps for several levels of hierarchy were chosen interactively, and the results leading to the best classification are shown. 
Table 3: Global classification accuracies in percentage for the Indiana image: Overall Accuracy (OA), Average Accuracy (AA) and kappa coefficient $(\kappa)$.

\begin{tabular}{|c|c|c|c|c|c|}
\hline \multicolumn{3}{|c|}{ Method } & $\overline{\mathrm{OA}}$ & $\overline{\mathrm{AA}}$ & $\kappa$ \\
\hline \multicolumn{3}{|c|}{ Pixel-wise SVM } & 78.76 & 69.66 & 75.73 \\
\hline \multirow{7}{*}{$\begin{array}{c}\text { SVM + } \\
\text { Majority Vote }\end{array}$} & \multirow{3}{*}{ No WHEDs } & SumBands & 87.03 & 75.24 & 85.18 \\
\hline & & Sum4PCA & 86.92 & 77.79 & 85.05 \\
\hline & & RCMG & 86.79 & 79.36 & 84.88 \\
\hline & \multirow{4}{*}{$\frac{\text { With WHEDs }}{\mathrm{HSE}}$} & SumBands & 93.78 & 80.53 & 92.88 \\
\hline & & Sum4PCA & 91.67 & 80.61 & 90.47 \\
\hline & & RCMG & 92.48 & 77.26 & 91.39 \\
\hline & & HSEG & 92.20 & 80.49 & 91.06 \\
\hline
\end{tabular}

As in the previous experiment, the combined spectral-spatial classification improved the classification accuracies, and the With WHEDs approach gave the best performances. The use of the watershed map based on the SumBands gradient led to the highest overall accuracy (an improvement by 15.02 percentage points compared to the pixel-wise classification) and kappa coefficient, while the highest average accuracy was achieved by using the Sum $4 P C A$ gradient (it is 10.95 percentage points higher than for the pixel-wise classification). The watershed based on the $R C M G$ gradient led to lower classification accuracies than the one based on the SumBands gradient. And the $R C M G$ approach gave a higher overall accuracy but a lower average accuracy than the Sum $4 P C A$ approach.

The class-specific accuracies were improved by using the spatial information in the classification for almost all the classes (see Table 4). After the combined spectral-spatial classification, the classification accuracy was reduced for only one class (oats). The classification using the With WHEDs approach led to 0\% accuracy for this class. The oats class has only 20 pixels in the reference map, and represents a small and very narrow (of 2 pixels width) rectangular field. Thus, after majority voting within the watershed regions, pixels of the oats class were assigned to the classes in its neighborhood (mostly to the grass/trees class). This drawback of spectral-spatial classification, i.e., the risk of loosing small spatial structures, was discussed in the previous subsection. 


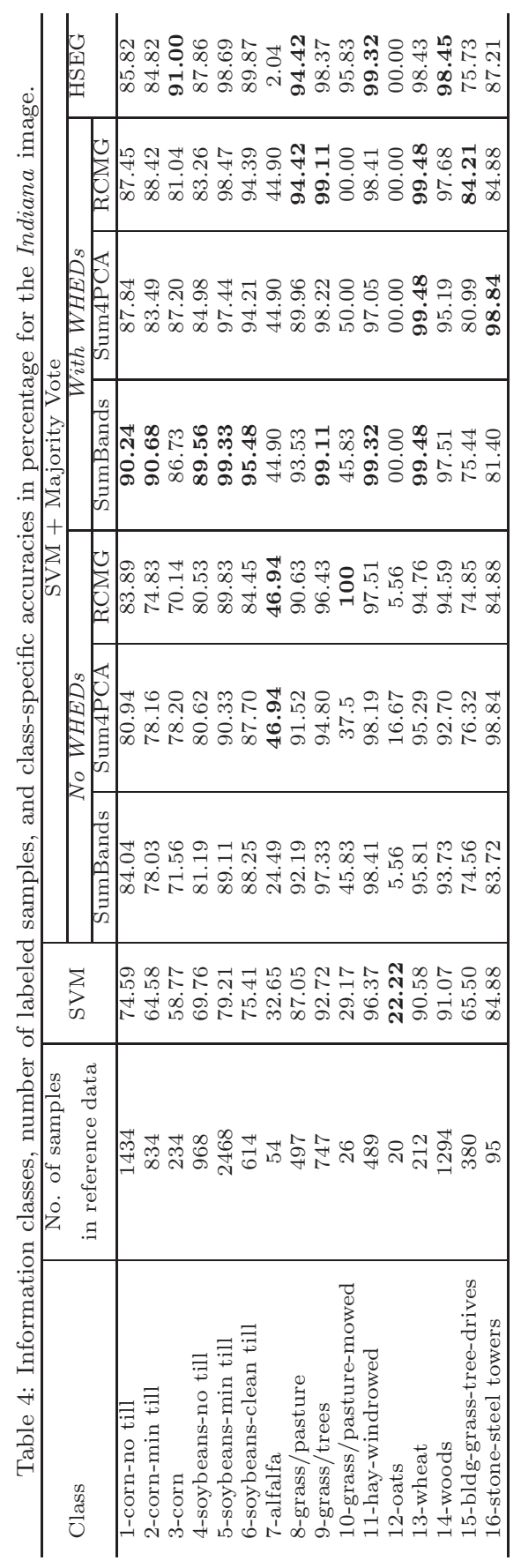




\subsubsection{Concluding discussion}

In assessing the watershed segmentation approaches we note that the results for the Indiana image are different from those for the University of Pavia image. For the Indiana image, the linear combination (sum) of gradients of all bands or several principal components leads to better segmentation results than the use of the vectorial gradient. This can be explained by the fact that the Indiana image contains more spectral channels (220 channels versus 103 channels for the University of Pavia image). Furthermore, the vectorial gradient, based on the Euclidean distance between pixel vectors does not give accurate results due to the curse of dimensionality. In this case, it appears to be more appropriate to compute marginal gradients of every band and to sum them together or to reduce the spectral dimension by performing feature extraction first, and then compute a gradient.

The spectral-spatial classification improved classification accuracies when compared to pixel-wise classification. The improvement of the global accuracies was more significant than for the University of Pavia image. From a visual observation (see Figures 11.(b) and 11.(c)), the classification map obtained by the spectral-spatial classification is seen to be much less noisy than the one obtained by the pixel-wise classification. From the obtained results, we can draw the conclusion that the Indiana image contains a lot of relatively large regularly structured spatial objects (crop fields) which is the reason why the inclusion of the spatial information was very efficient and improved the classification accuracies significantly.

If we analyze classification results obtained by majority vote within the HSEG regions, we can conclude that:

1. The global and most of class-specific accuracies are improved when compared to pixel-wise classification. This proves the efficiency of the proposed spectral-spatial classification scheme.

2. The accuracies are lower than those obtained when using watershed for segmentation (except for 2 classes). This confirms why it is desirable to 
use watershed segmentation for hyperspectral images.

\section{Conclusions}

Hyperspectral imaging provides rich spectral information per pixel, increasing the capability to distinguish physical structures in a scene. However, a large number of spectral channels presents challenges to image classification. While pixel-wise classification techniques process each pixel independently without considering information about spatial structures, further improvement of classification performances can be achieved by the incorporation of spatial information into classifier, especially in areas where structural information is important to distinguish between classes.

In this paper, there are two main contributions:

1. The extension of the watershed segmentation algorithm for hyperspectral images was proposed. In particular, different ways were investigated to obtain a one-band gradient function from a hyperspectral image and the combination of watershed segmentation maps was proposed.

2. A new methodology, a spectral-spatial classification scheme for hyperspectral images was proposed. The new method is based on the pixel-wise SVM classification, followed by majority voting within the watershed regions. Thus, segmentation defines an adaptive neighborhood for each pixel.

In two experiments it was shown that the combined spectral-spatial classification, based on majority voting within the regions obtained by the watershed segmentation algorithms, led to higher classification accuracies when compared to pixel-wise classification or previously proposed techniques. Furthermore, classification maps with more homogeneous regions were obtained with the proposed approach.

In conclusion, the proposed classification methodology succeeded in taking advantage of spatial the spectral information simultaneously. Furthermore, experimental results have revealed that it is desirable to use the watershed segmentation map in a spectral-spatial classifier. The watershed transformation is a 
completely unsupervised method since it does not require any input parameters (thresholds). Therefore, it can be incorporated into an automatic classification system.

The proposed spectral-spatial classification scheme is especially suitable in classifying images with large spatial structures. The drawback of the proposed method is that when applying watershed segmentation, small spatial structures are often not identified as separate regions. This leads to the assimilation of these structures with larger neighboring structures when majority voting is performed within the watershed regions.

In our future work, we will attempt to improve the segmentation results. In particular, we envision to explore the use of additional filtering and merging of regions for that purpose.

\section{Acknowledgments}

This research is supported in part by the Marie Curie Research Training Network "HYPER-I-NET". The authors would like to thank Paolo Gamba and

David Landgrebe for providing the hyperspectral data and James Tilton for providing the NASA Goddard's RHSEG software.

\section{References}

[1] C.-I. Chang, Hyperspectral Imaging: Techniques for Spectral Detection and Classification, Kluwer Academic Publishers, Dordrecht, 2003.

[2] R. O. Green, M. L. Eastwood, C. M. Sarture, T. G. Chrien, M. Aronsson, B. J. Chippendale, J. A. Faust, B. E. Pavri, C. J. Chovit, M. S. Solis, M. R. Olah, O. Williams, Imaging spectroscopy and the airborne visible infrared imaging spectrometer (AVIRIS), Remote Sens. Environ. 65 (3) (1998) 227-248.

[3] G. Hughes, On the mean accuracy of statistical pattern recognizers, IEEE Trans. Information Theory 14 (1) (1968) 55-63.

[4] D. Landgrebe, Hyperspectral image data analysis, IEEE Signal Proces. Mag. 1053-5888 (2002) 17-28. 
[5] D. A. Landgrebe, Signal Theory Methods in Multispectral Remote Sensing, John Wiley \& Sons, Inc., 2003.

[6] C. H. Chen, P.-G. Peter Ho, Statistical pattern recognition in remote sensing, Pattern Recognition 41 (9) (2008) 2731-2741.

[7] N. Bali, A. Mohammad-Djafari, Bayesian approach with Hidden Markov modeling and mean field approximation for hyperspectral data analysis, IEEE Trans. Image Processing 17 (2) (2008) 217-225.

[8] S. Subramanian, N. Gat, M. Sheffield, J. Barhen, N. Toomarian, Methodology for hyperspectral image classification using novel neural network, in: Proc. of SPIE, Vol. 3071, 1997, pp. 128-137.

[9] H. Yang, F. V. D. Meer, W. Bakker, Z. J. Tan, A back-propagation neural network for mineralogical mapping from AVIRIS data, International Journal of Remote Sensing 20 (1) (1999) 97-110.

[10] C. Hernández-Espinosa, M. Fernández-Redondo, J. Torres-Sospedra, Some experiments with ensembles of neural networks for classification of hyperspectral images, in: ISNN (1), 2004, pp. 912-917.

[11] P. K. Goel, S. O. Prasher, R. M. Patel, J. A. Landry, R. B. Bonnell, A. A. Viau, Classification of hyperspectral data by decision trees and artificial neural networks to identify weed stress and nitrogen status of corn, Comput. Electron. Agricult. 39 (2003) 67-93.

[12] H. Zhou, Z. Mao, D. Wang, Classification of coastal areas by airborne hyperspectral image, in: Proc. of SPIE, Vol. 5832, 2005, pp. 471-476.

[13] C. Vaiphasa, Innovative genetic algorithm for hyperspectral image classification, in: Proc. of the International Conference Map Asia, Vol. 20, 2003.

[14] G. Camps-Valls, L. Bruzzone, Kernel-based methods for hyperspectral image classification, IEEE Trans. Geos. and Remote Sens. 43 (6) (2005) 13511362 . 
[15] M. Fauvel, Spectral and spatial methods for the classification of urban remote sensing data, Ph.D. thesis, Grenoble Institute of Technology (2007).

[16] M. Fauvel, J. Chanussot, J. A. Benediktsson, Evaluation of kernels for multiclass classification of hyperspectral remote sensing data, in: Proc. of ICASSP'2006, Vol. 2, 2006, pp. II-813 - II-816.

[17] B. Guo, S. R. Gunn, R. I. Damper, J. D. B. Nelson, Customizing kernel functions for SVM-based hyperspectral image classification, IEEE Trans. Image Processing 17 (4) (2008) 622-629.

[18] M. Pesaresi, J. A. Benediktsson, A new approach for the morphological segmentation of high-resolution satellite imagery, IEEE Trans. Geos. and Remote Sens. 39 (2) (2001) 309-320.

[19] A. Farag, R. Mohamed, A. El-Baz, A unified framework for map estimation in remote sensing image segmentation, IEEE Trans. Geos. and Remote Sens. 43 (7) (2005) 1617-1634.

[20] K. Fu, J. Mui, A survey on image segmentation, Pattern Recognition 13 (1) (1981) 3-16.

[21] Y. Tarabalka, J. Chanussot, J. A. Benediktsson, J. Angulo, M. Fauvel, Segmentation and classification of hyperspectral data using watershed, in: Proc. of IGARSS'08, Boston, USA, 2008, pp. III-652 - III-655.

[22] Y. Tarabalka, J. A. Benediktsson, J. Chanussot, J. Angulo, M. Fauvel, Classification of hyperspectral data using Support Vector Machines and adaptive neighborhoods, in: Proc. of the 6th EARSeL SIG IS workshop, Tel Aviv, Israel, 2009.

[23] A. Darwish, K. Leukert, W. Reinhardt, Image segmentation for the purpose of object-based classification, Proc. of IGARSS'03, Vol. 3, 2003, pp. 20392041. 
[24] J. Tilton, Analysis of hierarchically related image segmentations, IEEE Workshop on Advances in Techniques for Analysis of Remotely Sensed Data (2003) 60-69.

[25] P. Soille, Morphological partitioning of multispectral images, Journal of Electronic Imaging 5 (3) (1996) 252-265.

[26] G. Flouzat, O. Amram, S. Cherchali, Spatial and spectral segmentation of satellite remote sensing imagery using processing graphs by mathematical morphology, Proc. of IGARSS '98, Vol. 4, 1998, pp. 1769-1771.

[27] P. Scheunders, Multivalued image segmentation based on first fundamental form, in: Proc. of the 11th International Conference on Image Analysis and Processing, 2001, pp. 185-190.

[28] P. Li, X. Xiao, Evaluation of multiscale morphological segmentation of multispectral imagery for land cover classification, Proc. of IGARSS'04, Vol. 4, 2004, pp. 2676-2679.

[29] G. Noyel, J. Angulo, D. Jeulin, Morphological segmentation of hyperspectral images, Image Analysis \& Stereology 26 (2007) 101-109.

[30] H. G. Akçay, S. Aksoy, Automatic detection of geospatial objects using multiple hierarchical segmentations, IEEE Trans. Geos. and Remote Sens. 46 (7) (2008) 2097-2111.

[31] J. Chanussot, P. Lambert, Bit mixing paradigm for multivalued morphological filters, in: Proc. of IEE IPA'97, 1997, pp. 804-808.

[32] J. Chanussot, P. Lambert, Total ordering based on space filling curves for multivalued morphology, in: Proc. of ISMM'98, 1998, pp. 51-58.

[33] P. Lambert, J. Chanussot, Extending mathematical morphology to color image processing, in: Proc. of CGIP'00, 2000, pp. 158-163.

[34] A. G. Hanbury, J. Serra, Morphological operators on the unit circle, IEEE Trans. Image Processing 10 (12) (2001) 1842-1850. 
[35] J. Angulo, J. Serra, Morphological coding of color images by vector connected filters, in: Proc. of ISSPA'2003, Vol. 1, 2003, pp. 69-72.

[36] E. Aptoula, S. Lefèvre, A comparative study on multivariate mathematical morphology, Pattern Recognition 40 (11) (2007) 2914-2929.

[37] L. Shafarenko, M. Petrou, J. V. Kittler, Automatic watershed segmentation of randomly textured color images, IEEE Trans. Image Processing 6 (11) (1997) 1530-1544.

[38] J. Chanussot, P. Lambert, Watershed approaches for color image segmentation, in: Proc. of IEEE NSIP'99, 1999, pp. 129-133.

[39] J. Angulo, J. Serra, Mathematical morphology in color spaces applied to the analysis of cartographic images, in: Proc. of the GEOPRO 2003 - International Workshop Semantic Processing of Spatial Data, 2003, pp. 59-66.

[40] J. Angulo, Unified morphological color processing framework in a lum/sat/hue representation, in: Proc. of ISMM'2005, 2005, pp. 387-396.

[41] S. V. der Linden, A. Janz, B. Waske, M. Eiden, P. Hostert, Classifying segmented hyperspectral data from a heterogeneous urban environment using support vector machines, Journal of Applied Remote Sensing 1 (1, 013543) (2007).

[42] A. Widayati, B. Verbist, A. Meijerink, Application of combined pixel-based and spatial-based approaches for improved mixed vegetation classification using IKONOS, in: Proc. of the 23th Asian conference on remote sensing, 2002, 8 p.

[43] R. Cook, I. McConnell, C. J. Oliver, MUM (Merge Using Moments) segmentation for SAR images, in: Proc. of SPIE., Vol. 2316, 1994, pp. 92-103.

[44] P. Soille, Morphological Image Analysis, 2nd Edition, Springer-Verlag, 2003. 
[45] AVIRIS NW Indianas Indian Pines 1992 data set [Online]. Available: ftp://ftp.ecn.purdue.edu/biehl/MultiSpec/92AV3C (original files) and ftp://ftp.ecn.purdue.edu/biehl/PC MultiSpec/ThyFiles.zip (ground truth).

[46] F. Meyer, S. Beucher, Morphological segmentation, Journal of Visual Communication and Image Representation 1 (1990) 21-46.

[47] S. Beucher, C. Lantuejoul, Use of watersheds in contour detection, in Int. Workshop Image Processing, Real-Time Edge and Motion Detection/ Estimation. Rennes, France: CCETT/IRISA (1979).

[48] L. Vincent, P. Soille, Watersheds in digital spaces: an efficient algorithm based on immersion simulations, IEEE Trans. Pattern Analysis and Machine Intel. 13 (6) (1991) 583-598.

[49] F. Meyer, Topographic distance and watershed lines, Signal Process. 38 (1) (1994) 113-125.

[50] S. Stoev, Rafsi - a fast watershed algorithm based on rainfalling simulation, in: Proc. of the 8th International Conference on Computer Graphics, Visualization, and Interactive Digital Media, 2000.

[51] V. Osma-Ruiz, J. I. Godino-Llorente, N. Sáenz-Lechón, P. Gómez-Vilda, An improved watershed algorithm based on efficient computation of shortest paths, Pattern Recognition 40 (3) (2007) 1078-1090.

[52] J. A. Richards, X. Jia, Remote Sensing Digital Image Analysis: An Introduction, Springer-Verlag New York, Inc., 1999.

[53] M. R. Gupta, N. P. Jacobson, Wavelet Principal Component Analysis and its application to hyperspectral images, in: Proc. of IEEE ICIP'06, 2006, pp. 1585-1588.

[54] A. A. Green, M. Berman, P. Switzer, M. D. Craig, A transformation for ordering multispectral data in terms of image quality with implications for noise removal, IEEE Trans. Geos. and Remote Sens. 26 (1) (1988) 65-74. 
[55] J. Wang, C.-I. Chang, Independent component analysis-based dimensionality reduction with applications in hyperspectral image analysis, IEEE Trans. Geos. and Remote Sens. 44 (6) (2006) 1586-1600.

[56] S. Moussaoui, H. Hauksdottir, F. Schmidt, C. Jutten, J. Chanussot, D. Brie, S. Dout'e, J. A. Benediktsson, On the decomposition of Mars hyperspectral data by ICA and Bayesian positive source separation, Neurocomputing 71 (2008) 2194-2208.

[57] A. N. Evans, X. U. Liu, A morphological gradient approach to color edge detection, IEEE Trans. Image Processing 15 (6) (2006) 1454-1463.

[58] J. Astola, P. Haavisto, Y. Neuvo, Vector median filters, Proc. of the IEEE 78 (4) (1990) 678-689.

[59] ITT Corporation, For further information about the ENVI software: http://rsinc.com/envi/.

[60] C. Chang, C. Lin, LIBSVM - a library for support vector machines, Software available at http://www.csie.ntu.edu.tw/ cjlin/libsvm (2008).

[61] A. Plaza, J. A. Benediktsson, J. Boardman, J. Brazile, L. Bruzzone, G. Camps-Valls, J. Chanussot, M. Fauvel, P. Gamba, J. A. Gualtieri, M. Marconcini, J. C. Tilton, G. Trianni, Recent advances in techniques for hyperspectral image processing, Remote Sensing of Environment. 113 (Supplement 1) (2009), pp. S110-S122.

[62] J. A. Benediktsson, J. A. Palmason, J. R. Sveinsson, Classification of hyperspectral data from urban areas based on extended morphological profiles, IEEE Trans. Geos. and Remote Sens. 43 (3) (2005) 480-491.

[63] S. Aksoy, Spatial techniques for image classification, in: C. H. Chen, ed., Signal and Image Processing for Remote Sensing, Taylor \& Francis, (2006), pp. 491-513. 
[64] M. Fauvel, J. Chanussot, J. A. Benediktsson, J. R. Sveinsson, Spectral and spatial classification of hyperspectral data using SVMs and morphological profiles, IEEE Trans. Geos. and Remote Sens. 46 (10) (2008).

[65] S. Tadjudin, D. Landgrebe, Classification of high dimensional data with limited training samples, Ph.D. thesis, School of Electrical and Computer Engineering, Purdue University (1998).

[66] J. C. Tilton, HSEG/RHSEG, HSEGViewer and HSEGReader user's manual (version 1.40), Provided with the evaluation version of RHSEG available from: http://ipp.gsfc.nasa.gov/RHSEG. 